\title{
Littéracie, SMS et troubles spécifiques du langage écrit
}

\author{
Thi Mai Tran ${ }^{1,2}$, Marine Trancart $^{2} \&$ Domitille Servent ${ }^{2}$ \\ ${ }^{1}$ UMR 8163 « Savoirs, textes, Langage » \\ Université de Lille 3 \\ BP 60149 \\ 59653 Villeneuve d'Ascq Cedex \\ ${ }^{2}$ Institut d'Orthophonie \\ Faculté de Médecine \\ 59045 Lille Cedex \\ thimai.tran@univ-lille 2.fr
}

\section{Introduction}

La fin du XXème siècle a été marquée par un essor sans précédent des Nouvelles Technologies de l'Information et de la Communication (NTIC). Courriels, forums de discussion, blogs, SMS, tchats sont autant de situations nouvelles de communication écrite nées du développement et de la diffusion des outils électroniques (ordinateur et téléphone mobile). L'utilisation de plus en plus étendue de ces outils, que ce soit dans le cadre privé, professionnel voire scolaire, est à l'origine de nouveaux codes et de manières différentes de traiter l'écrit. De fait, les conditions techniques de la communication électronique ont une influence sur les pratiques scripturales et le rapport que les individus entretiennent avec la langue écrite.

Investies dans le domaine de la pathologie du langage, nous nous sommes demandées si ces nouveaux outils, devenus en l'espace de quelques années quasi incontournables, étaient investis de la même manière par les personnes présentant des troubles du langage écrit dans le cadre de pathologies primaires (troubles spécifiques du langage écrit : Trancart \& Servent, 2006) ou de pathologies secondaires (surdité : Laporte \& Le Galloudec, 2006). Notre questionnement porte sur la possibilité, pour les personnes présentant des difficultés particulières et durables dans les situations d'écrits traditionnels, d'accéder aux NTIC et le cas échéant, les modalités de cet accès. Il s'intéresse aux compétences linguistiques et cognitives nécessaires à la maîtrise des SMS et aux pratiques sociales et culturelles liées à ce type d'écrit. Il se situe dans le champ de la littéracie (Jaffré, 2004).

L'étude que nous présentons se limitera à la pratique des SMS chez des adolescents dyslexiques/dysorthographiques. Après avoir défini le support SMS et les troubles spécifiques du langage écrit, nous détaillerons l'expérience sur les écrits SMS que nous avons menée auprès de 24 adolescents. Nous discuterons ensuite ses principaux résultats en focalisant plus particulièrement sur les processus cognitifs mis en jeu dans le traitement de ce type d'écrit.

\section{Le cadre de l'étude}

\section{$2.1 \quad$ Les SMS}

\subsubsection{Keske C ?}

La messagerie SMS (Short Message Service ou texto en français) est un service de la téléphonie mobile qui a connu depuis sa création en 1992 un développement exponentiel. On compte actuellement en France 53 millions d'utilisateurs : $78 \%$ des foyers sont équipés d'un téléphone portable, le taux d'équipement des jeunes utilisateurs étant particulièrement important ( $70 \%$ chez les $12-14$ ans, $89 \%$ chez les $15-17$ ans et $95 \%$ chez les $18-25$ ans). $70 \%$ des détenteurs de téléphone portable utilisent des SMS avec un envoi moyen de 25 SMS par mois ce qui correspond à un marché de 21 milliards d'euros en 2007 (sources : 
Afom 2007). Chez les 12-25 ans, ce chiffre monte jusqu'à plus de 80 SMS par mois témoignant de l'engouement indéniable des jeunes pour ce mode de communication écrite.

Le SMS est un message écrit de 160 caractères maximum envoyé d'un téléphone portable à un autre. Sa forme linguistique et graphique est contrainte par différents facteurs : techniques (espace limité, clavier alpha-numérique), économiques (coût du SMS) et situationnels (type d'informations échangées et interlocuteurs concernés). Ces petits messages sont la plupart du temps envoyés à une personne proche pour communiquer une information courte et pratique, poser rapidement une question ou partager en quelques mots ou signes une émotion, un sentiment ou un point de vue. Compte-tenu des caractéristiques de l'outil, le but pour le scripteur est de produire un message intelligible le plus court possible afin de réduire le coût d'encodage du message (nombre de pressions digitales) et le coût de sa transmission (prix de la communication). Pour les utilisateurs experts, c'est un moyen de communication rapide (potentiellement quasi-instantané), efficace (l'information principale étant donnée de manière directe), discret (absence de sonnerie et d'oralisation) et économique (moins coûteux qu'un appel téléphonique). Pour les collégiens et les lycéens, la possession d'un téléphone portable et la maîtrise de l'écrit SMS sont devenus des signes d'appartenance au groupe générationnel, à ses codes, un moyen de transgresser la norme sociale (ici l'écriture conventionnelle des adultes). Outil de socialisation favorisant l'échange entre pairs, il constitue également moyen d'émancipation : comme le souligne Pasquier (2005), ce mode de communication échappe la plupart du temps au contrôle parental et fait l'objet par les adolescents d'une gestion très habile. C'est sans doute pour ces multiples raisons que l'écrit SMS rencontre un tel succès auprès des 12-25 ans.

\subsubsection{Caractéristiques de l'écriture SMS}

L'écriture SMS se définit d'une part, par un principe général d'économie des signes grâce à l'utilisation d'abréviations et de procédés phonologiques permettant d'utiliser un nombre de lettres réduit et d'autre part, par un ancrage de la langue écrite dans la langue orale. La communication médiée par la téléphonie (CMT) est à l'origine d'une écriture spécifique (Anis, 2001 ; Liénard 2005a) possédant des règles à la fois propres et instables, une grande liberté étant laissée, au final, au scripteur.

Le principe d'économie est à l'origine de réductions et de simplifications des formes écrites (à devenant «a », habit: «abi », veste: « vest», veux : «v», clair : «kler», qui : «ki », beau : «bo », forfait: « forfè », père : «per » ou encore vous venez : "vs vené ») ainsi que le recours à un recodage du mot ou d'une partie de celui-ci sur des critères phonologiques (manger devenant «manG», demander: « $2 \mathrm{manD} »$ ou cité : «6T »).

L'oralité imprègne la plupart du temps les messages SMS, que ce soit au niveau phonologique, lexical, syntaxique ou encore au niveau du découpage de la suite de signes (qu'est ce que tu fais ? : «kestufé? », t'as pas vu: «tapavu? », à tout à l'heure: «ataleur », je suis : « chuis », je sais pas : « chépa ») comme si le message était de l'oral écrit. C'est ce que Liénard (2005) nomme le parler écrit ou «parlécrit ». Les caractéristiques suprasegmentales de la parole sont notées par des procédés graphiques spécifiques: étirements de signes pour signifier l'allongement vocalique ("viiiiiiite ») ou réitérations de signes ou usages de majuscule pour marquer l'intensité («JE T’AIME», «j’tador!!!!!!!!»). Les éléments non verbaux peuvent être signifiés par des symboles propres à la communication électronique et inventés dans ce cadre (émoticônes, smileys : «:)» pour $\odot$ ou « : (» pour $(:)$ ). Le recours à des anglicismes ou des siglaisons codées achève de donner une touche originale au message ( mdr » pour mort de rire ou « kiss » pour bises).

Les SMS offrent, par rapport à l'écrit traditionnel, une liberté et une souplesse d'utilisation. Le scripteur dispose, dans sa transcription, de plusieurs choix. Il peut personnaliser son message, varier le nombre et le type de procédés utilisés en fonction de son humeur et de son interlocuteur. L'instabilité, la pluralité des formes mais également la polysémie sont une conséquence de cette latitude laissée au scripteur : un mot peut être écrit par un sujet de plusieurs façons dans un même message ou dans des messages différents («à bientôt », «a biento » « abiento », « abil to »...) et une même suite de signes peut référer à plusieurs 
significations («slt»: salut ou seulement)... La prise en compte de cette variabilité des formes écrites complique la tâche du lecteur qui doit développer de nouvelles compétences en matière de déchiffrage.

Cette écriture abrégée, phonétique et expressive, éloignée à bien des égards de la norme orthographique, suscite des réactions parfois extrêmes allant de l'engouement à l'aversion. Certains y voient une forme d'écriture originale et créative qui enrichit les expériences scripturales à l'heure où on reproche souvent aux jeunes générations de désinvestir l'écrit au profit de l'audio-visuel. D'autres, une dénaturation et un appauvrissement de la langue écrite condamnables et dangereux. Depuis les années 2000, la littérature ou la toile font appel à de nouvelles dénominations pour désigner ces nouvelles pratiques écrites : langage SMS , sociolecte écrit, néolangage, cyberécriture, cyber@langue (Dejong, 2002). Des ouvrages en « SMS » voient le jour (Marso 2004). Sociologues et linguistes s'intéressent de plus en plus cette forme d'écrit maintenant bien installée dans la génération des moins de 25 ans (Anis 1999, Jaffré 2003, Pasquier 2005, Liénard 2005a , Fairon \& al. 2006).

Selon nous, l'écrit SMS correspond à une nouvelle variété du français écrit liée à un support particulier dans le cadre d'interactions communicatives déterminées. Cette variété est la résultante de combinaison originale de procédés scripturaux multiples, connus depuis longtemps et utilisés dans différents domaines : jeux (rébus), prise de notes (abréviations), littérature, chanson, publicité...Cet écrit concerne des messages du quotidien. Ces messages sont souvent informels et familiers (expression moins contrôlée que dans les courriers manuscrits), immédiats (écrits bruts nécessitant peu ou pas de relecture) et affectifs (l'interlocuteur est souvent proche et partage un certain degré de connivence avec le scripteur). Les dimensions phatique et expressive du message sont parfois aussi importantes (voire plus importantes) que la dimension référentielle : le message SMS est un moyen de maintenir un contact, de partager des émotions. Chez les adolescents, l'appropriation de ce moyen de communication joue un rôle de marqueur d'identité (Liénard, 2005c). Le caractère ludique, non conventionnel et non normatif de l'écriture SMS rencontre une adhésion forte de cette population, raison pour laquelle nous avons choisi de nous intéresser à cette catégorie de sujets.

\subsection{Les troubles spécifiques du langage écrit}

\subsubsection{La dyslexie}

On estime à environ $20 \%$ la proportion d'élèves en difficultés de lecture à l'entrée au collège. Ces difficultés concernent les capacités de décodage et/ou de compréhension du langage écrit. Elles peuvent être associées ou pas à d'autres difficultés plus générales.

Selon le rapport Ringard (2000), parmi ces élèves, la proportion d'élèves présentant des troubles spécifiques du langage écrit se réduirait à 4 à $6 \%$. Dans ce même rapport, les troubles spécifiques sévères conduisant à des situations de handicap et nécessitant des aménagements pédagogiques particuliers sont estimés à $1 \%$ des élèves scolarisés. Le terme de trouble spécifique du langage écrit y est utilisé de manière préférentielle afin de mettre l'accent sur le fait que le trouble touche de manière spécifique les mécanismes d'identification des mots écrits chez des enfants intelligents et ayant des capacités d'expression et de compréhension orale normales. Les troubles de l'acquisition de la lecture s'accompagnent de troubles dans l'acquisition et la maitrise de l'orthographe. Le terme de dyslexie, même s'il est le plus souvent utilisé seul, recouvre une réalité plus large correspondant cliniquement à une atteinte du traitement réceptif et productif de la langue écrite (dyslexie/dysorthographie).

Il existe plusieurs types de troubles spécifiques du langage écrit (Carbonnel \& al., 1996 ; Estienne \& Van Hout 2001) mais on s'accorde à considérer que les troubles phonologiques sont la plupart du temps au premier plan dans cette pathologie (Génard \& al., 1994 ; Sprenger Charolles \& al., 2000). Même si les sujets s'expriment habituellement avec aisance à l'oral, ils rencontrent des difficultés particulières dans le traitement explicite des unités de la langue (phonèmes et graphèmes) et ont des performances déficitaires dans les épreuves métaphonologiques (ex. : identification de rimes, repérage d'un phonème ou d'un groupe de phonèmes dans un mot, répétition de logatomes, opérations de suppression, ajout et/ou 
inversion de phonèmes). Ces difficultés spécifiques sont, souvent décrites sous le terme de troubles de la conscience phonologique, sont caractérisés par un déficit dans la manipulation intentionnelle des phonèmes. Ces capacités étant mobilisées dans la lecture et l'écriture des messages SMS, nous nous sommes donc interrogées sur la possibilité pour les jeunes dyslexiques de s'approprier ce type d'outil.

\subsubsection{Caractéristiques de la population étudiée}

Notre population d'étude se compose de 24 collégiens et lycéens ayant une pratique régulière des SMS. Elle comprend 12 jeunes dyslexiques âgés de 14 à 20 ans appariés à 12 jeunes non dyslexiques de même sexe, âge et originaires du même milieu socioculturel avec un équilibre des sexes (12 filles et 12 garçons). L'ensemble des sujets est originaire de Versailles et de sa périphérie.

Les 12 adolescents de la population pathologique présentent des difficultés durables et spécifiques dans la maitrise et l'automatisation des mécanismes de lecture associés à une dysorthographie correspondant à un tableau de troubles spécifiques du langage écrit. Pour tous ces sujets, un diagnostic de dyslexie a été posé au cours de la scolarité primaire et a été suivi d'une rééducation orthophonique (toujours en cours au moment de l'expérience). Les performances en lecture et en orthographe de notre population dyslexiques (élèves scolarisés de la $4^{\text {ème }}$ à la terminale) mesurées par le Test de l'alouette (Lefavrais, 1965) et la dictée de Vaney (Frerré, 1961) correspondent à des performances d'élèves du primaire (CE1 à CM2) ce qui confirme un décalage important de leurs compétences à l'écrit par rapport à la norme et à la population contrôle. Aucun des sujets de ce groupe ne présente de troubles sensoriels, intellectuels ou psychoaffectifs. Ils proviennent de milieux ordinaires à favorisés et ont suivi d'une scolarisation régulière. Leurs principales caractéristiques sont résumées dans le tableau 1 ci-dessous (afin de préserver l'anonymat des sujets, tous les prénoms ont été modifiés).

Tableau 1 : Caractéristiques générales de la population dyslexique

\begin{tabular}{|c|c|c|c|c|}
\hline \multirow{2}{*}{ PRENOM } & \multirow{2}{*}{ AGE } & \multirow{2}{*}{ CLASSE } & \multicolumn{2}{|c|}{ NIVEAU DE LECTURE } \\
\cline { 4 - 5 } & & $4^{\text {ème }}$ & AGE & CLASSE \\
\hline Valentin & 14 ans & $5^{\text {ème }}$ & 9 ans & CE2 \\
\hline Jérôme & 14 ans & $4^{\text {ème }}$ & 10 ans & CM1 \\
\hline Sabine & 14 ans & $3^{\text {ème }}$ & 11 ans & CM2 \\
\hline Morgane & 14 ans & $2^{\text {nde }}$ & 10 ans & CM2 \\
\hline Mattéo & 15 ans & $3^{\text {ème }}$ & 10 ans & CM2 \\
\hline Françoise & 15 ans & $3^{\text {ème }}$ & 8 ans & CE2 \\
\hline Justine & 15 ans & $3^{\text {ème }}$ & 10 ans & CM2 \\
\hline Anselme & 15 ans & $3^{\text {ème }}$ & 8 ans & CE2 \\
\hline David & 15 ans & BEP & 9 ans & CM1 \\
\hline Florent & 16 ans & CAP & 10 ans & CM1 \\
\hline Cécile & 18 ans & Tle STI & 8 ans & CE2 \\
\hline Arthur & 20 ans & & &
\end{tabular}


ISBN 978-2-7598-0358-3, Paris, 2008, Institut de Linguistique Française

Psycholinguistique, acquisition

DOI $10.1051 / \mathrm{cmlf0} 034$

Les 12 adolescents non dyslexiques ont des résultats scolaires dans la moyenne, n'ont jamais été suivi en orthophonie et ont une moyenne générale supérieure ou égale à 11/20. 


\subsection{Problématique}

Partant des caractéristiques des troubles spécifiques du langage écrit d'une part, et des particularités de l'écriture SMS d'autre part, nous sommes posées deux types de questions.

Premièrement, considérant que les dyslexiques ont, dans plupart des cas, des compétences phonologiques déficitaires et que ces compétences sont nécessaires à l'utilisation de certains procédés fréquents dans l'écrit SMS, on peut se demander si ces sujets ne rencontrent pas de difficultés particulières dans la lecture et/ou l'écriture de SMS. Pour répondre à cette première question, nous avons proposé à nos deux groupes de sujets (dyslexiques et non dyslexiques) deux tâches de lecture de SMS dans le but de comparer leurs performances respectives (qualité du déchiffrage, temps de lecture, le nombre et le type d'erreurs, compréhension du message).

Deuxièmement, considérant que l'écriture SMS, dans son souci d'économie, simplifie à la fois l'orthographe lexicale et grammaticale et qu'elle n'impose pas de règles strictes, on peut se demander si ce mode de communication écrite ne faciliterait pas, cette fois, la production écrite des dyslexiques. Nous avons donc également proposé aux deux groupes deux tâches d'écriture de SMS et comparé leurs productions (intelligibilité du message, rapidité d'écriture, nombres et types de procédés utilisés, nombre et types d'erreurs).

\section{Le protocole}

Notre protocole comprend deux situations complémentaires : une épreuve de lecture et une épreuve d'écriture de SMS. Il explore deux types de traitement de l'écrit : la production et la compréhension. Dans la tâche de lecture, les procédés SMS utilisés sont imposés au lecteur qui est contraint d'effectuer un traitement des formes proposées pour pouvoir lire le message. En revanche, dans la tâche de dictée, le choix des procédés scripturaux est laissé au sujet.

\subsection{L'élaboration du protocole}

Préalablement à construction des épreuves du protocole, nous avons effectué un relevé des différents types de procédés habituellement utilisés dans l'écriture SMS à partir des classifications élaborées par Anis (2001) et Liénard (2005a) et réalisé une enquête sur les habitudes communicationnelles des jeunes en la matière.

L'enquête réalisée auprès de 41 collégiens et lycéens nous a permis de répertorier les pratiques (ex. : utilisation préférentielle du mode $\mathrm{ABC}$ ) et les thèmes d'échanges sur ce support. Elle a également fait apparaître que seuls, certains des sujets dyslexiques interrogés (au nombre de 9 parmi les 41 élèves ayant répondu à notre questionnaire), se plaignaient d'avoir parfois des difficultés à lire des messages qui leur étaient envoyés ou à se faire comprendre dans leurs écrits. Aucune plainte de ce type n'était formulée par les autres élèves.

Le recueil des principaux procédés d'écriture SMS résumés dans le tableau 2 nous a servi de base à la construction du matériel expérimental. 
Tableau 2. Les principaux procédés SMS

\begin{tabular}{|c|c|c|}
\hline CiBLES & CODAGES SMS & PROCEDES \\
\hline pas, font, dis & pa, fon, di & élision de la consonne finale muette \\
\hline lumière, laisse & Lumièr, laiss & élision de la voyelle finale \\
\hline Etienne, Elle & Etienne, elle & élision de la majuscule \\
\hline à, espère & $\mathrm{a}$, espere & élision de l'accent \\
\hline s'évaporer, s'en & sévaporer, sen & élision de l'apostrophe \\
\hline on y va & onyva & suppression de l'espace inter-mots \\
\hline minute, toute & $\min$, tte & abréviation \\
\hline nous, dans, salut & $\mathrm{ns}, \mathrm{ds}$, slt & abréviation consonantique \\
\hline je suis & chuis & écrasement de signe \\
\hline quoi, parler & koi, parlé & réduction de digramme consonantique \\
\hline $\mathrm{au}$ & o & réduction de digramme vocalique \\
\hline est & é & réduction de trigramme vocalique \\
\hline en & an & remplacement de digramme vocalique \\
\hline joie & jwa & remplacement d'un trigramme vocalique \\
\hline c'est, j'ai, ouvert, copain & $\mathrm{C}, \mathrm{G}$, ouvR, cop 1 & notation sémio-phonologique \\
\hline moins, à plus &,- à + & logogramme \\
\hline fête & teuf & verlan \\
\hline coucou & kikou & expressivité orale \\
\hline mort de rire & $\mathrm{mdr}$ & siglaison \\
\hline bises & kiss & anglicisme \\
\hline
\end{tabular}

Plusieurs critères ont été retenus lors de l'élaboration des textes écrits servant de support à la tâche de lecture et d'écriture. Nous avons d'abord essayé de prendre en compte le maximum de procédés décrits. Nous avons ensuite considéré deux niveaux de langage (familier et soutenu). Enfin, nous avons tenté d'intégrer un critère de complexité cognitive des procédés utilisés dans les SMS.

\subsubsection{Langage familier et le langage soutenu}

Nous avons construit deux messages correspondant aux thématiques et au niveau de langage (familier) habituellement associés aux messages SMS : l'un pour la situation de lecture, l'autre la situation d'écriture. Nous avons ainsi demandé aux sujets de lire et d'écrire des messages SMS adressés à un ami au sujet d'un rendez-vous et d'une fête. Ces deux textes ont été composés de telle sorte que les différents procédés répertoriés soient présents dans le message. 
Le message écrit à lire était le suivant: Coucou Pierre! Comment ça va? J'ai appris aujourd'hui que tu faisais une fête demain soir chez toi. Où est-ce que c'est? J'aimerais bien passer avec un copain, t'es ok j'espère? De toute façon, je viendrai quand même. Mort de rire, Mille bisous soit sur l'écran du téléphone portable présenté aux sujets :

\section{Lecture de SMS (langage familier)}

Kikou PiR! koman
sava? G apri ojrdui ke
tu fEzé 1 teuf $2 \mathrm{~m} 1$
swar ché twa
Weskesé ? Gmeré

bil pa $\mathrm{C}$ av 1 cop1 $\mathrm{T}$ ok GspR ? 2 tte fason $\mathrm{j}$ vildré qd mèm lol. 1000 bsx

Dans le cas de la dictée, le message proposé comprenait plus de 160 caractères afin d'induire un codage SMS accompagné, au besoin d'une consigne ludique, pour obtenir la réduction du nombre de caractères par rapport au message cible proposé («Malheureusement tu n'as plus suffisamment de forfait pour envoyer deux textos, est-ce que tu peux reprendre ce message pour qu'il ne fasse plus qu'un texto »). Les sujets écrivaient donc sous dictée sur leur portable le message suivant :

\section{Dictée SMS (langage familier)}

Salut Etienne Qu'est ce tu fais ? T'as oublié le rendez vous ou quoi ? Je suis à la cité avec ma roeus. On t'attend c'est relou. Dis nous au moins si tu viens ou pas au ciné. Si t'es pas là dans 10 minutes on y va. A plus.

A cette situation «classique », nous avons ajouté une situation de langage plus élaboré ne correspondant pas aux écrits habituellement échangés dans les messages SMS. Notre objectif était de confronter les jeunes à un lexique et une syntaxe différents pour évaluer dans quelle mesure ceux-ci étaient capables de recourir à des procédés SMS sur un matériel linguistique plus complexe et moins familier. Nous avons ainsi sélectionné deux passages de poèmes : en lecture, il s'agissait d'un extrait de G. Apollinaire et en dictée de P. Eluard.

\section{Lecture de SMS (langage soutenu)}

Ss le pon mirabo koul la $\mathrm{sN}$ é no amour fotil kil men souviN la jwa vené tjs aprè lé $\mathrm{pN}$ viN la nui sone l'h lé jrs

sen von j 2 meur lé $\mathrm{m} 1$ ds lé $\mathrm{ml}$ reston fas a fas tandi ke pass dé zéternL regar lon2 si lass apolinR 
correspondant à : Sous le pont Mirabeau coule la Seine et nos amours faut-il qu'il m'en souvienne? La joie venait toujours après les peines. Vienne la nuit, sonne l'heure. Les jours s'en vont, je demeure. Les mains dans les mains, restons face à face, tandis que sous le pont de nos bras, passent des éternel regards, l'onde si lasse. Apollinaire

\section{Dictée SMS (langage soutenu)}

Elle a toujours les yeux ouverts et ne me laisse pas dormir. Ses rêves en pleine lumière font s'évaporer les soleils, me font rire, pleurer et rire, parler sans avoir rien à dire. Paul Eluard.

\subsubsection{Complexité psycholinguistique des procédés}

Afin de déterminer la nature et la complexité des procédés utilisés, nous nous sommes appuyées sur les principes de l'écriture alphabétique (Jaffré \& Fayol, 1997) et sur les processus de traitement des mots écrits (Ellis, 1990). En effet selon ces auteurs, d'une part, l'écriture alphabétique repose sur un double principe : le principe phonographique permettant de noter les unités sonores de la langue et le principe sémiographique permettant de noter les unités significatives de la langue. D'autre part, le traitement cognitif des mots écrits ferait appel à deux types de processus (l'adressage et l'assemblage) et à deux voies (la voie phonologique et la voie lexicale). La voie phonologique recourrait à des opérations de conversion graphème $\leftrightarrow$ phonème correspondant à la procédure d'assemblage. Cette voie serait utilisée au début de l'apprentissage et ensuite, chez le lecteur habile, essentiellement pour déchiffrer les mots inconnus. La voie lexicale permettrait de récupérer de manière directe, à partir du sens, la forme phonologique ou graphémique du mot par le biais de la procédure d'adressage. Cette dernière impliquerait un traitement visuel de l'information écrite s'appuyant sur les connaissances orthographiques stockées en mémoire (dans le lexique orthographique) tandis que la voie phonologique reposerait davantage sur un traitement auditif faisant appel à des opérations de conversions entre unités de la langue orale et unités de la langue écrite. Ellis (1990) parle de «lecture auditive » pour la lecture par voie phonologique et de « lecture visuelle» pour la lecture par voie lexicale. Ces deux types de lecture peuvent être mis en correspondance avec les principes phonographiques et sémiographiques de l'écriture française précédemment cités.

Sur cette base, nous avons distingués deux types de procédés illustrés dans le tableau 3 :

les procédés faisant appel aux aspects auditifs du traitement de la langue écrite et relevant du principe phonographique (recours à la procédure d'assemblage et à la conversion graphème $\leftrightarrow$ phonème) ;

les procédés faisant appel aux aspects visuels du traitement de la langue écrite et relevant du principe sémiographique (recours à la procédure d'adressage et au lexique orthographique). 
Tableau 3 : Classification psycholinguistique des procédés SMS

\begin{tabular}{|c|c|c|}
\hline $\begin{array}{c}\text { TYPES DE } \\
\text { PROCEDES }\end{array}$ & PRINCIPE & ILLUSTRATION \\
\hline $\begin{array}{c}\text { Procédés } \\
\text { phonographiques } \\
\text { SMS }\end{array}$ & $\begin{array}{c}\begin{array}{c}\text { Economie de signes } \\
\text { dans }\end{array} \\
\text { la correspondance } \\
\text { graphème } \leftrightarrow \text { phonème }\end{array}$ & 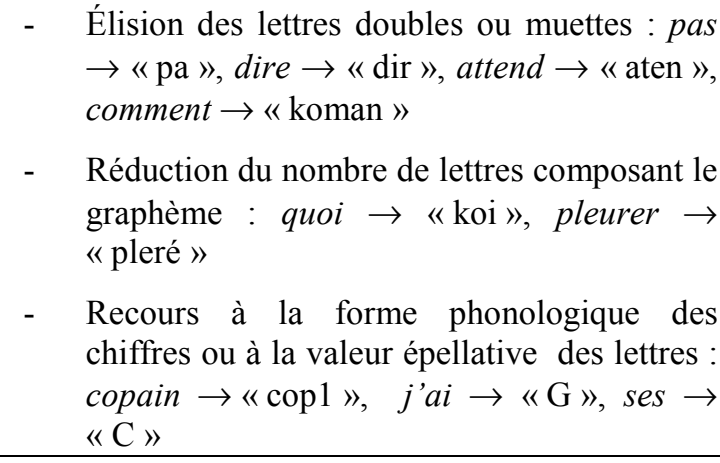 \\
\hline $\begin{array}{c}\text { Procédés } \\
\text { sémiographiques } \\
\text { SMS }\end{array}$ & $\begin{array}{l}\text { Economie de signes } \\
\text { dans l'écriture du ou } \\
\text { des mot(s) }\end{array}$ & $\begin{array}{l}\text { - Elision de signes : majuscules, espaces, } \\
\text { accents, ponctuation: on y va } \rightarrow \text { "onyva }, \\
\grave{a} \rightarrow \text { a », qu'est ce que tu fais } \rightarrow \\
\text { «kestufé » } \\
\text { - Réduction du nombre de lettres composant le } \\
\text { mot: avoir } \rightarrow \text { «avr», salut } \rightarrow \text { slt }, \\
\text { rendez-vous } \rightarrow \text { rv » }\end{array}$ \\
\hline
\end{tabular}

Les procédés phonographiques-SMS reposent sur le principe général d'économie par réduction du nombre de lettres contenues dans le graphème lors des correspondances graphème $\leftrightarrow$ phonème (ou encore forme phonologique $\leftrightarrow$ lettre /chiffre). Ils s'appuient sur la forme phonologique (ou auditive) du mot écrit. Selon ce principe, les lettres muettes sont élidées, les graphèmes comprenant deux à trois lettres sont réduits à une ou deux lettres (ex. : qu $\rightarrow$ « $\mathrm{k} »$, ain $\rightarrow$ « in », eau $\rightarrow$ « $\mathrm{o} »)$ et la valeur épellative des lettres ou la forme phonologique des chiffres sont utilisées pour coder, à la manière des rébus, les mots écrits (ex. : j'ai $\rightarrow$ «G», cité $\rightarrow$ «ciT» ou «6T», un $\rightarrow$ « $1 »$ ). Ces procédés supposent des compétences phonologiques (connaissance des correspondances graphème $\leftrightarrow$ phonème, de la valeur épellative des lettres). Leur application aboutit à une écriture phonétique : la compréhension du message écrit nécessite alors un passage par la voie phonologique. L'oralisation ou la sub-vocalisation du message permet d'accéder à son sens ( « tu v1 o 6né »= tu viens au ciné).

Les procédés sémiographiques-SMS s'appuient sur la forme orthographique (ou visuelle) des mots : les opérations de réduction s'appliquent à cette forme et visent à ne conserver que les éléments nécessaires à l'identification visuelle du message. Ces procédés supposent une connaissance orthographique initiale du mot à simplifier. La reconnaissance s'effectue visuellement et la forme réduite est récupérée par adressage.

Parmi ces deux types de procédés, certains peuvent être considérés comme plus complexes que d'autres. En effet, les procédés de réduction ou de simplifications (ex. : «pa », « slt », «koi ») font appel à des opérations simples de suppression ou de remplacement de signes alors les opérations de recodage utilisées dans les procédés sémiophonologiques (ex. : «G», «Ojourd8») mobilisent davantage de représentations (phonologiques, graphémiques et sémantiques) et de processus cognitifs. 
Enfin, et c'est souvent le cas, ces procédés peuvent être combinés entre eux dans la transcription d'une unité lexicale ou propositionnelle (ex. : «Cvaporé » pour s'évaporer «si T pala » pour si t'es pas là).

\section{Résultats}

D'un point de vue général, les résultats de notre étude ne font pas apparaître d'écart important entre les performances du groupe de dyslexiques et du groupe de non dyslexiques.

Du point de vue du type et du nombre de procédés utilisés, les performances de nos deux populations dans les épreuves de lecture et surtout d'écriture de SMS sont globalement comparables. Au vu du corpus, il est la plupart du temps difficile d'identifier le statut du scripteur (dyslexique ou non dyslexique). La dyslexie ne semble donc pas constituer un obstacle majeur à la pratique des SMS. Du point de vue de l'écriture des messages, il semble que le support SMS soit de nature à faciliter la production des sujets dyslexiques.

L'analyse qualitative des réponses permet, en revanche, de pointer des différences entre les deux populations. A titre d'illustration, nous présentons dans le tableau 4 des exemples de corpus recueillis au cours de notre étude afin de visualiser ces différences.

Tableau 4 : exemples de SMS produits en situation de dictée

Salut Etienne, Qu'est ce tu fais? T'as oublié le rendez vous ou quoi? Je suis à la cité avec ma roeus. On t'attend c'est relou. Dis nous au moins si tu viens ou pas au ciné. Si t'es pas là dans 10 minutes on y va. A plus.

\begin{tabular}{|c|c|}
\hline sujets non dyslexiques & sujets dyslexiques \\
\hline
\end{tabular}

- Salu etiene kestu fai? ta ou blie rdv ou koi?chui ala cite ac ma reuss on taten $\mathrm{c}$ relou.di nou au moin si tu v1 o cine,si t pa la dan 10 min on yva $a^{+}$

- Salu etiene kestu fai ta oublié le rdv ou koi je sui a la cité ac ma roes on taten c relou di ns o moin si tu vien ou pa o ciné si t pa la ds 10 min on $\mathrm{i}$ va a+
- Slt etién ké t fé ta oubli je rdv ou k je si a a la sit ac ma res on tatan c rlu di ns $m$ moi si $t$ vin ou pa $m$ siné si $t$ pal ds 10 min on ni v aps

- $\quad$ slt tien $t$ fe ko t oubli rdevou ko chi la site av m seur o tn c rlou di ns $\mathrm{s} t$ vien o cin $\mathrm{s} t$ po $1 \mathrm{ds} 10 \mathrm{~min}$ on iva pluse

Elle a toujours les yeux ouverts et ne me laisse pas dormir. Ses rêves en pleine lumière font s'évaporer les soleils, me font rire, pleurer et rire, parler sans avoir rien à dire. Paul Eluard. sujets non dyslexiques sujets dyslexiques 
- El a tjr les yeu ouver et ne me laiss pa dormir.c rev en pleine lumier fon sevaporer ls soleil me fon rir, pleurer et rir,parler san avoir r1 a diq.paul eluard

- El a tjs lé yeu ouver et ne me laiss pa dormir c reve en pleine lumier fon sèvaporè lé soleil me fon rir pleuré é rir parlé ss avoir rien a dire paul eluar
- El a tjr ls ie ouvr e m léj s pa drmir c rév an plén lmiér fon cévaporé ls soly me fon rr plré é rr parlé sn avr ril a dir q pléluar

- el tjour 1 ye vert m me les p dormir c reve an plein lumier fo vapore 1 soliel $\mathrm{m}$ fe lol chial $\mathrm{t}$ lol parle san voir $\mathrm{r} 1 \mathrm{dir}$ pol t luar

En réception des messages, les dyslexiques ont une lecture plus lente que les non dyslexiques (leur vitesse de lecture est en moyenne deux fois plus faible). En écriture, ils continuent de produire des erreurs spécifiques comme des inversions (ex. : «soliel» pour soleil, «Maribeau » pour Mirabeau), des confusions de phonèmes (ex. : «vin » pour viens, « renté » pour rendez), des erreurs de correspondance forme graphique $\leftrightarrow$ forme phonique (ex. : «étaine » pour Etienne, « a la » pour elle a, «g $»$ pour je), de découpage de mots (ex. : «on ni » pour on y, « ou blie» pour oublié). Ils utilisent parfois des réductions non pertinentes (« $\mathrm{k} »$ pour quoi ou « sn » pour sans ou «tn» pour t'attend) ou complexifient le codage au point que le mot n'est plus identifiable (phénomène spécifique à la population pathologique dans notre étude). Ces difficultés apparaissent essentiellement dans les situations de langage soutenu (extraits de poèmes) et pour les procédés phonologiquement complexes (ex. : « GspR », « viN » ou « lon2»).

Globalement, dans les SMS écrits par la population dyslexique, les difficultés spécifiques liées à pathologie restent présentes. Il s'agit des difficultés dans le traitement phonologique (à l'origine de notre questionnement) mais également de difficultés dans le traitement visuel en particulier liées à l'instabilité et/ou à la pauvreté du lexique orthographique des sujets dyslexiques. Nous avons moins insisté sur ce point dans notre présentation initiale des troubles du langage écrit mais nos résultats, en particulier dans les tâches de lecture, montrent que cet aspect du traitement des mots écrits est également à prendre en compte pour expliquer les performances de nos sujets pathologiques.

Si ces difficultés demeurent présentes, il est important de souligner, au terme de ce travail, que celles-ci sont moins importantes et moins invalidantes dans les messages SMS que dans les situations d'écrit traditionnel. En écriture, ces difficultés sont minimisées en raison des contraintes moins importantes de l'écriture SMS. En revanche, en lecture, les caractéristiques de l'écriture SMS, en particulier l'instabilité des formes graphiques et la présence de procédés de type phonographiques, peuvent pénaliser la lecture des sujets dyslexiques.

5 Discussion Avant de conclure, un résultat étonnant par rapport à nos hypothèses initiales est à relever. Comme indiqué supra, les sujets dyslexiques de notre étude utilisent l'ensemble des procédés à leur disposition et en particulier les procédés phonographiques complexes (ex. : «G », «Ojourd8 », « tu v1 o 6né »). A première vue, cette observation va à l'encontre de notre hypothèse de difficultés d'accès à ces procédés en raison du dysfonctionnement de la voie phonologique dans la dyslexie.

Après un nouvel examen de nos corpus, nous formulons l'hypothèse suivante : la taille réduite du lexique orthographique et des unités graphiques utilisés dans les messages SMS favoriserait le développement de stratégies de type sémiographiques. Dans cette perspective, certaines formes complexes phonologiquement pourraient être traitées par la voie lexicale (c'est-à-dire directement récupérées par adressage) sans passer par une médiation phonologique. La pratique des SMS conduirait à l'élaboration 
d'un «lexique orthographique SMS » où seraient stockées les unités graphiques mémorisées suite à l'utilisation réitérée de ces unités.

Le format, par définition réduit, des unités utilisées dans les SMS faciliterait leur mémorisation. Cette hypothèse semble confirmée par le fait que certains de nos sujets dyslexiques interrogés étaient capables d'accéder au sens et à la forme phonologique du mot lu, en lecture et en écriture (ex. : «Ojourd8》" aujourd'hui), sans pour autant pouvoir expliciter les mécanismes phonologiques à l'origine du codage de ces mêmes mots. Le fait que les difficultés des dyslexiques soient particulièrement repérables dans la situation de langage soutenu, c'est-à-dire lorsqu'on leur demande des traiter des unités moins familières, est un argument de plus dans ce sens.

\section{Conclusion}

Ce travail exploratoire montre que les adolescents dyslexiques de notre population utilisent, au même titre que leurs pairs, l'écrit SMS. Le caractère moins contraignant et simplificateur de l'écriture SMS mais aussi le nombre souvent limité des unités linguistiques utilisées dans les messages via la téléphonie mobile, sont de nature à faciliter l'appropriation de ce code écrit par les sujets dyslexiques : en lecture, ils peuvent utiliser des stratégies de compensation de type sémiographiques et en écriture, leurs difficultés se trouvent moins stigmatisées comme l'indique les propos d'un jeune dyslexique de notre étude : «j'aime écrire des SMS car on ce comprend, il n'y à pas de sujements sur l'hortographe». Ce nouveau mode de communication rend possible un rapport «décomplexé » à l'écrit et participe à l'intégration du jeune dyslexique dans le groupe de ses pairs.

Ce résultat positif est toutefois à nuancer si on considère le cadre limité dans lequel peut s'exercer cette pratique scripturale. Parmi l'ensemble des situations de traitement de l'écrit auxquelles sont confrontés les collégiens et lycéens, au quotidien ou dans leur scolarité, l'écrit SMS semble cantonné à un cadre précis (interlocuteur familier, thématiques simples, niveau d'élaboration linguistique restreint) et pourrait être réduit à une pratique minoritaire.

Dans les faits, cette pratique est relayée par d'autres supports que la téléphonie mobile comme l'ordinateur (MSN, blogs, tchats...). Pour les premières générations de la communication électronique, cette pratique est venue enrichir des compétences écrites déjà installées, l'accès aux outils informatiques et au téléphone portable se faisant ultérieurement, souvent au collège ou au lycée. La plupart des jeunes issus de cette génération, sont capables d'appréhender les normes de l'écrit en fonction des situations et des interlocuteurs (ex. : distinction entre l'écrit scolaire et l'écrit MSN, SMS adressé aux adultes dans un écrit normatif). L'écriture SMS est pour eux un moyen d'expression supplémentaire dont ils maîtrisent les codes notamment sociaux. Cependant, à l'heure où les enfants sont de plus en plus tôt en contact avec les nouvelles technologies, on est en droit de s'interroger sur les effets à long terme d'une pratique précoce et régulière des SMS et autres écritures électroniques, non seulement chez des sujets en difficultés d'apprentissage et d'utilisation fonctionnelle du langage écrit et mais aussi chez les enfants en phase d'acquisition de langage écrit.

\section{Références}

Anis J. (éd), (1999), Internet, communication et langue française, Paris : Hermes Science Public.

Anis J. (2001), Parlez vous texto ?, Paris : éd. Le cherche-midi.

Carbonnel S., Gillet P., Matory M.-P. \& Valdois S. (1996), Approche cognitive des troubles de la lecture et de l'écriture chez l'enfant et l'adulte, Marseille : Solal.

Ellis A.W. (1990), Lecture, écriture et dyslexie : une approche cognitive, éd. Delachaux Niéstlé.

Estienne F., Van Hout A. (2001), Les Dyslexies : décrire, évaluer, expliquer et traiter, Paris : Masson.

Fairon C.,Klein J-R., Paumier S. (2006) Le langage SMS; Etude d'un corpus informatisé à partir de l'enquête "Faites don de vos SMS à la science », UCL Presses Universitaires de Louvain 
Genard N., Mousty P., Alegria J. (2004), « Troubles phonologiques et sous-types de la dyslexie du développement», in Valdois S., Cole P., David D. (dir) (2004). Apprentissage de la lecture et dyslexies développementales : de la théorie à la pratique orthophonique et pédagogique. Marseille : Solal, 147-170.

Jaffre J.-P, Fayol M., (1997), Orthographes des systèmes aux usages, Evreux : Dominos Flammarion.

Jaffre J.-P. (2003), «L'écriture et les nouvelles technologies : ce que les unes nous apprennent de l'autre » in : Réseaux humains/réseaux technologiques, S'écrire avec les outils d'aujourd'hui, MSHS/CRDP de PoitouCharente, 4, 81-86 [Office audiovisuel de l'université de Poitiers, http://imedias.univ-poitiers.fr/rhrt/index.htm]

Jaffre J.-P. (2004), «La littéracie : histoire d'un mot, effets d'une notion » in Barre de Miniac C., Brissaud C., Rispail M. (eds), La littéracie. Conceptions théoriques et pratiques d'enseignement de la lecture-écriture. Paris: L'Harmattan, 21-41

Jaureguiberry F. (2003), Les branchés du portable, Paris : PUF.

Laporte A., Le Galloudec M. (2006), Les adolescents sourds et le SMS : une nouvelle pratique de l'écrit pour mieux communiquer?, Mémoire d'orthophonie, Université de Lille 2.

Lienard F. (2005a), « Analyse linguistique et sociopragmatique d'une pratique scripturale particulière » in Berube J., Gauvin K., Remysen W (dir.), Actes du $18^{\mathrm{e}}$ colloque Les journées de linguistique, Université de Laval Québec, éd. Ciral , 79-92.

Lienard F. (2005b), «SMS : une menace pour le français ? », Cerveau \& Psycho, n¹2, 28-31.

Lienard Fabien (2005c). «La construction identitaire virtuelle en CMO et CMT »,Internet-Zeitschrift fûr Kulturwissenschaften, Trans $\mathrm{n}^{\circ} 16$, http://www.inst.at/trans/16Nr/11 1/lienard16.htm

Marso P. (2004), SMS Pa Sage a taba vo SMS, Editions Megacom-ik.

Pasquier D. (2005), Cultures lycéennes La tyrannie de la majorité, Paris : éd. Mutations.

Ringard J.-C. (2000), "A propos de l'enfant «dysphasique » et de l'enfant «dyslexique » », Rapport à Mme la ministre déléguée à l'enseignement scolaire, http://www.education.gouv.fr/cid1944/a-propos-de-l-enfantdysphasique-et-de-l-enfant-dyslexique.html.

Sprenger-Charolles L., Lacert P., Cole P. (1999), «Déficits phonologiques et métaphonologiques chez des dyslexiques phonologiques et de surface », Rééducation orthophonique $\mathrm{n}^{\circ} 197$.

Trancart M., Servent D. (2006) : Dyslexiques et SMS : Komen S kil fonksione?, Mémoire d'orthophonie, Université de Lille 2. 